\title{
Utilization of arsenic trioxide as a treatment of cisplatin-resistant non-small cell lung cancer PC-9/CDDP and PC-14/CDDP cells
}

\author{
TOSHIHIRO SUZUKI $^{1}$, KENICHI ISHIBASHI ${ }^{1}$, ATSUSHI YUMOTO ${ }^{1}$, KAZUTO NISHIO $^{2}$ and YUKI OGASAWARA ${ }^{1}$ \\ ${ }^{1}$ Department of Analytical Biochemistry, Meiji Pharmaceutical University, Kiyose, Tokyo 204-8588; \\ ${ }^{2}$ Department of Genome Biology, Kinki University School of Medicine, \\ Osaka-sayama, Osaka 589-8511, Japan
}

Received August 26, 2014; Accepted May 20, 2015

DOI: $10.3892 / 01.2015 .3352$

\begin{abstract}
Cisplatin is a commonly used drug in combination chemotherapy. However, various malignant tumors frequently acquire resistance to cisplatin. Arsenic trioxide (ATO) has been approved as a chemotherapeutic drug for the treatment of acute promyelocytic leukemia, and the combination of ATO and cisplatin has been revealed to demonstrate synergistic effects in ovarian and small cell lung cancer cells. Thus, it was hypothesized that ATO may also be active against cisplatin-resistant non-small cell lung cancer (NSCLC) PC-9/CDDP and PC-14/CDDP cells. The present study also evaluated the effects of ATO on the cisplatin-sensitive NSCLC PC-9 and PC-14 cell lines. Notably, ATO demonstrated a markedly decreased $\mathrm{IC}_{50}$ in the cisplatin-resistant PC-9/CDDP and PC-14/CDDP cells compared with the $\mathrm{IC}_{50}$ in the cisplatin-sensitive parental PC-9 and PC-14 cells. Additionally, it was found that arsenite accumulation in the PC-9 cell line was affected through the downregulation of GS-X pump systems. Although it is likely that cisplatin resistance in PC-9 cells does not depend on the GS-X pump systems, ATO was effective against cisplatin-resistant NSCLC PC-9/CDDP and PC-14/CDDP cells in combination chemotherapy.
\end{abstract}

\section{Introduction}

Since the late 20th century, a large number of molecular targeted therapeutic drugs, such as epidermal growth factor receptor tyrosine kinase inhibitors, have been developed for cancer chemotherapy. However, platinum-based combination chemotherapy continues to be important for the

Correspondence to: Professor Toshihiro Suzuki, Department of Analytical Biochemistry, Meiji Pharmaceutical University, 2-522-1 Noshio, Kiyose, Tokyo 204-8588, Japan

E-mail: tssuzuki@my-pharm.ac.jp

Key words: cisplatin resistance, arsenic trioxide, glutathione, adenosine triphosphate-binding cassette subfamily $\mathrm{C}$ transporter treatment of various cancers, including lung cancer. Cisplatin is a commonly used drug for combination chemotherapy (1). Although cisplatin is a potent anticancer drug, various malignant tumors frequently acquire resistance to cisplatin, limiting the clinical application of this agent $(2,3)$.

Arsenite is a toxic metalloid that is widely distributed in the environment (4). Despite the toxicity, arsenic-containing compounds have been used in traditional Chinese medicine for $>2,000$ years (5). In addition, arsenic trioxide (ATO) has been approved in numerous countries for the treatment of acute promyelocytic leukemia (APL) (6). In vitro studies have revealed that a combination of ATO and cisplatin exerts a synergistic inhibitory effect on the growth of ovarian cancer and small cell lung cancer cells (7-9).

It is well known that adenosine triphosphate-binding cassette $(A B C)$ transporters are important molecules for the resistance of cancer cells to chemotherapy agents and the detoxification of toxic metals in xenobiotic metabolism (10). Leslie et al previously reported that $\mathrm{ABC}$ subfamily $\mathrm{C}$, member 1 (ABCC1) and ABC, subfamily $\mathrm{C}$, member 2 (ABCC2) each play an important role in multidrug resistance and arsenic detoxification in cells, through the cellular efflux of the glutathione (GSH) conjugate (11).

The present study aimed to examine the sensitivity of the non-small cell lung cancer (NSCLC) PC-9 cell line and cisplatin-resistant PC-9/CDDP subline to toxic metals. The cisplatin-resistant PC-9/CDDP cell line demonstrated hypersensitivity to arsenite and arsenate. Furthermore, the accumulation of arsenite was decreased in PC-9/CDDP cells relative to the parental cells. Notably, the sensitivity to arsenite and accumulation of arsenite were markedly increased compared with the cisplatin-resistant cell line. Therefore, it was hypothesized that the GS-X pump systems were downregulated in the cisplatin resistant NSCLC cell line. In addition, the arsenic-containing chemotherapeutic agent ATO was applied to the cisplatin-sensitive and -resistant NSCLC cells, and it was found that the cisplatin-resistant NSCLC cell lines PC-9/CDDP and PC-14/CDDP were hypersensitive to ATO.

The results of the present study suggest that arsenite accumulation depends on the GS-X pump and that treatment with ATO may overcome certain cases of cisplatin resistance in NSCLC. 


\section{Materials and methods}

Cell lines and materials. The NSCLC PC-9 and PC-14 cell lines, which were derived from untreated patients with pulmonary adenocarcinoma, were provided by Professor Y. Hayata (Tokyo Medical College, Tokyo, Japan). The cisplatin-resistant PC-9/CDDP and PC-14/CDDP sublines were established at the National Cancer Center Research Institute (Tokyo, Japan) (12).

The mouse anti-human ABCC1 monoclonal antibody (clone number, QCRL1; ALX-801-010) and mouse anti-human ABCC2 monoclonal antibody (clone number, M2I-4; ALX-801-015) were obtained from Enzo Life Sciences, Inc. (Farmingdale, NY, USA). The mouse anti-human GST- $\pi$ monoclonal antibody (clone number, GST- $\pi / 3$; 610718) was obtained from BD Biosciences (Franklin Lakes, NJ, USA). The Vectastain ABC kit and horseradish peroxidase-conjugated anti-mouse immunoglobulin $\mathrm{G}$ secondary antibody were obtained from Vector Laboratories (Burlingame, CA, USA). Enhanced chemiluminescence (ECL) reagent and Hyperfilm were obtained from GE Healthcare Biosciences (Pittsburgh, PA, USA). Sodium serenate, sodium selenite, sodium arsenite and sodium arsenate were obtained from Sigma-Aldrich (St. Louis, MO, USA). Antimony and cadmium chloride were obtained from Nacalai Tesque, Inc. (Kyoto, Japan). Arsenic trioxide (Trisenox) was obtained from Nippon Shinyaku Co. Ltd. (Kyoto, Japan). All other chemicals were obtained from Nacalai Tesque, Inc. or Wako Pure Chemical Industries, Ltd. (Osaka, Japan).

Cellular cytotoxicity assay to determine sensitivity to metals. Cell counting kit-8 was obtained from Dojindo Molecular Technologies, Inc. (Kumamoto, Japan) and, with MTT, was used for colorimetric assays to determine the cell viability, as previously described (13). The cells were plated on 96-well microplates at a density of $2 \times 10^{3}$ cells per well, incubated overnight, and then treated with various concentrations of arsenate, arsenite, selenate, selenite, mercury, cadmium or antimony for $72 \mathrm{~h}$.

Determination of arsenite accumulation. The cells were plated on $6-\mathrm{cm}$ dishes at a density of $1 \times 10^{6}$ cells per dish, incubated overnight, and then treated with $100 \mu \mathrm{M}$ sodium arsenite for $4 \mathrm{~h}$. Subsequent to washing with ice-cold PBS (-), the cells were immediately harvested using a cell scraper. The cells were pelleted by centrifugation, and the cell pellets were extracted with $20 \mathrm{mM}$ Tris- $\mathrm{HCl}(\mathrm{pH} 7.4)$ containing $1 \%$ Triton-X 100 and $0.1 \%$ SDS. The arsenite concentrations in the cells were determined by atomic absorption spectrometry (AAS; Z-9000; Hitachi, Ltd., Tokyo, Japan).

Analysis of the GSH concentration and activity of GSH-associated enzymes. The intracellular GSH concentration was measured using 5,5-dithiobis(2-nitrobenzoic acid) (DTNB). Cell lysates were diluted in $100 \mathrm{mM}$ sodium phosphate buffer ( $\mathrm{pH}$ 7.4) containing $2.5 \mathrm{mM}$ EDTA, $0.25 \mathrm{mM}$ NADPH and 1 unit/ml glutathione reductase (Oriental Yeast, Co., Ltd., Tokyo, Japan). The reaction was then initiated by adding $200 \mu \mathrm{M}$ DTNB, and the absorbance at $415 \mathrm{~nm}$ was subsequently measured. The amount of total GSH was determined by comparing the absorbance of the cell lysate with a GSH standard curve.
The activity of $\gamma$-glutamyl cysteine synthetase $(\gamma$-GCS) was measured using a previously reported high-performance liquid chromatography (HPLC) method (14), with slight modification. A $10-\mu \mathrm{l}$ aliquot of cell lysate was diluted in $100 \mu \mathrm{l}$ of assay mixture containing $75 \mathrm{mM} \mathrm{KCl}, 10 \mathrm{mM} \mathrm{MgCl}_{2}, 0.2 \mathrm{mM}$ EDTA, $10 \mathrm{mM}$ adenosine triphosphate, $10 \mathrm{mM}$ cysteine, $5 \mathrm{mM}$ glutamate and $100 \mathrm{mM}$ Tris- $\mathrm{HCl}$ buffer (pH 7.4). Following $20 \mathrm{~min}$ of incubation at $37^{\circ} \mathrm{C}$, the reaction was terminated by the addition of $20 \mu \mathrm{l}$ of $15 \%$ trichloroacetic acid, and the assay mixture was then chilled on ice. Subsequent to centrifugation, quantitation of Glu-Cys was conducted by post-labeled fluorescence detection HPLC using the ortho-phthalaldehyde reagent (Nacalai Tesqe, Inc., Kyoto,Japan).

Glutathione-S-transferase (GST) activity was determined using standard spectrophotometric assays by monitoring the formation of the conjugate of 1-chloro-2,4-dinitrobenzene (CDNB) and reduced glutathione (GSH) (15).

GST- $\pi$ protein expression was determined using immunoblotting. In total, $20 \mu \mathrm{g}$ of protein was loaded onto $12.5 \%$ (w/v) SDS-polyacrylamide gels and subjected to electrophoresis. Subsequent to electrotransfer onto polyvinylidene difluoride (PVDF) membranes (Bio-Rad Laboratories, Hercules, CA, USA), the membranes were blocked with Block Ace (Yukijirushi-Nyugyo, Co., Sapporo, Japan) according to the manufacturer's instructions. Probing was performed overnight at $4{ }^{\circ} \mathrm{C}$ with the monoclonal mouse anti-GST- $\pi$ antibody diluted with PBS containing 0.1\% Tween-20 (1:200; clone number, 3; BD Biosciences, Franklin Lakes, NJ, USA). Subsequent to washing, specific signals were detected using horseradish peroxidase-conjugated anti-mouse immunoglobulin $\mathrm{G}$ secondary antibody diluted with PBS containing $0.1 \%$ Tween-20 (1:10,000) and ECL reagent according to the manufacturer's instructions (GE Healthcare Biosciences, Pittsburgh, PA, USA).

$R N A$ extraction and reverse transcription-quantitative polymerase chain reaction $(R T-q P C R)$ for $m R N A$. Total RNA was isolated using an RNeasy Mini kit (Qiagen, Hilden, Germany). Reverse transcription was performed on $1 \mu \mathrm{g}$ of total RNA using High Capacity RNA-to-cDNA Master Mix (Applied Biosystems, Foster City, CA, USA). RT-qPCR was performed using converted cDNA and the following reagents: TaqMan Gene Expression Assays for human ABCC1 (assay ID, Hs00219905_m1), ABCC2 (assay ID, Hs00166123_m1), ABCC3 (assay ID, Hs00978473_m1), ABCC4 (assay ID, Hs00988717_m1), ABCC5 (assay ID, Hs00981087_m1) and GAPDH (assay ID, Hs99999901_s1) genes, and the TaqMan Universal PCR Master Mix, no AmpErase UNG (Thermo Fisher Scientific Inc., Waltham, MA). An ABI PRISM 7300 Sequence Detector System (Thermo Fisher Scientific Inc.) was used to collect the signals according to the manufacturer's instructions. The relative gene expression was determined using the comparative cycle threshold (CT) method based on the normalization of the target gene against the GAPDH reference gene. $\triangle \mathrm{CT}$ was calculated by subtracting the $\mathrm{CT}$ of GAPDH from the CT of the target genes.

Immunoblotting of $A B C C 1$ and $A B C C 2$. Plasma membrane fractions were prepared by ultracentrifugation, as previously described (16). Plasma membrane fractions (50 $\mu \mathrm{g}$ protein) 
Table I. Cross-sensitivity for various metals in the cisplatin-resistant PC-9/CDDP cell subline.

\begin{tabular}{|c|c|c|c|}
\hline \multirow[b]{2}{*}{ Metal compounds } & \multicolumn{2}{|c|}{$\mathrm{IC}_{50}, \mu \mathrm{M}$} & \multirow{2}{*}{$\begin{array}{l}\text { Relative } \\
\text { resistance }\end{array}$} \\
\hline & PC-9 & PC-9/CDDP & \\
\hline Antimony (IV) & $97.5 \pm 5.0$ & $25.0 \pm 10.0^{\mathrm{a}}$ & $0.26 \pm 0.10$ \\
\hline Arsenite (III) & $22.5 \pm 5.0$ & $3.3 \pm 1.3^{\mathrm{a}}$ & $0.14 \pm 0.03$ \\
\hline Arsenate (V) & $240.0 \pm 89.4$ & $19.0 \pm 2.2^{\mathrm{a}}$ & $0.09 \pm 0.02$ \\
\hline Selenite (IV) & $9.8 \pm 0.4$ & $9.8 \pm 0.4$ & $1.00 \pm 0.00$ \\
\hline Selenate (VI) & $140.0 \pm 54.8$ & $260.0 \pm 134.1$ & $1.90 \pm 0.55$ \\
\hline Mercury (II) & $92.5 \pm 9.6$ & $22.5 \pm 12.6$ & $0.25 \pm 0.14$ \\
\hline Cadmium (II) & $62.5 \pm 22.2$ & $70.0 \pm 21.6$ & $1.15 \pm 0.24$ \\
\hline Cisplatin (II) & $4.1 \pm 0.3$ & $23.8 \pm 4.8$ & $5.76 \pm 1.20$ \\
\hline
\end{tabular}

$\mathrm{IC}_{50}$, half maximal inhibitory concentration. ${ }^{\mathrm{a}} \mathrm{P}<0.05$ vs. PC-9 cells.

Table II. Concentration of GSH and activity of associated enzymes in the cisplatin-resistant PC-9/CDDP cell subline.

\begin{tabular}{lccc}
\hline Cell line & $\begin{array}{c}\text { GSH, } \\
\text { nmol/mg }\end{array}$ & $\begin{array}{c}\text { GCS, } \\
\text { unit/mg }\end{array}$ & $\begin{array}{c}\text { GST, } \\
\text { unit/mg }\end{array}$ \\
\hline PC-9 & 144.0 & 1.70 & 0.397 \\
PC-9/CDDP & 95.5 & 1.13 & 0.016 \\
Ratio, \% of PC-9 & 66.3 & 66.5 & 4.0 \\
\hline
\end{tabular}

GSH, glutathione; GCS, glutamyl cysteine synthetase; GST, glutathione-S-transferase.

Table III. Sensitivity to arsenic trioxide in the parent and cisplatin-resistant non-small cell lung cancer PC-9 and PC-14 cell lines.

\begin{tabular}{|c|c|c|c|}
\hline \multirow[b]{2}{*}{ Cell line } & \multicolumn{2}{|c|}{$\mathrm{IC}_{50}, \mu \mathrm{M}$} & \multirow{2}{*}{$\begin{array}{l}\text { Relative } \\
\text { resistance, } \\
\text { fold of parent }\end{array}$} \\
\hline & Parent & Cisplatin resistant & \\
\hline PC-9 & $5.6 \pm 1.3$ & $1.4 \pm 0.2$ & $0.3 \pm 0.1$ \\
\hline PC-14 & $4.5 \pm 2.7$ & $1.1 \pm 0.7$ & $0.3 \pm 0.1$ \\
\hline
\end{tabular}

$\mathrm{IC}_{50}$, half maximal inhibitory concentration.

were loaded onto $7.5 \%$ (w/v) SDS-polyacrylamide gels and resolved by electrophoresis. Following electrotransfer onto PVDF membranes, probing was performed overnight at $4^{\circ} \mathrm{C}$ with the anti-ABCC1 antibody or anti-ABCC2 antibody. Subsequent to washing, specific signals were detected using the Vectastain ABC system and ECL chemiluminescence reagent.

Statistical analysis. The results are expressed as the mean \pm standard deviation. Statistical evaluations were performed using Student's t-tests with Microsoft Excel 2013 (Microsoft Corporation, Redmond, WA, USA) for the analysis.

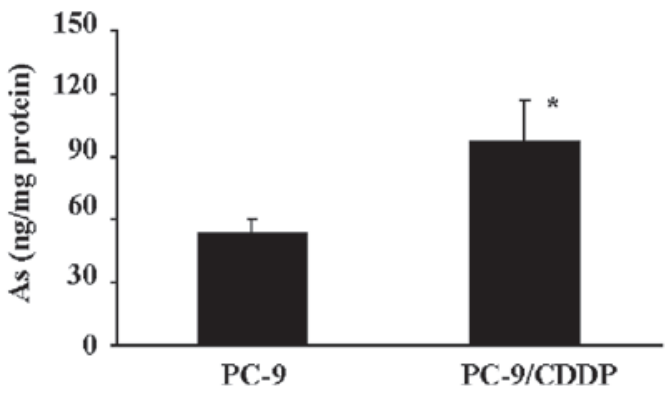

Figure 1. Accumulation of As in PC-9 and PC-9/CDDP cells. The accumulation of As in PC-9 and PC-9/CDDP cells was measured subsequent to incubation with $100 \mathrm{mM}$ sodium As for $4 \mathrm{~h}$. Bars represent the mean \pm standard deviation of triplicate independent experiments. ${ }^{*} \mathrm{P}<0.05$ vs. PC -9 cells. As, arsenite.

\section{Results}

Cross-resistance to metals. To elucidate the mechanism underlying cisplatin resistance, an analysis of the sensitivity of the PC-9 and PC-9/CDDP cell lines to various metals was performed. The $\mathrm{IC}_{50}$ values of various metal compounds in the parental PC-9 and cisplatin-resistant PC-9/CDDP cell lines are reported in Table I. Only selenate (VI) demonstrated an increased cross-resistance in PC-9/CDDP cells compared with parental PC-9 cells, with a 1.90-fold increase. By contrast, antimony (IV), arsenate (V), arsenite (III) and mercury (II) were revealed to be more potent inhibitors of PC-9/CDDP cells compared with the effect on the parental PC-9 cell line. No difference in sensitivity was observed for the other metals between the PC-9/CDDP and parental PC-9 cells.

Arsenite accumulation. The PC-9/CDDP cells demonstrated hypersensitivity to arsenite and arsenate. To elucidate the mechanisms of arsenite sensitivity in the cisplatin-resistant PC-9/CDDP cell line, the accumulation of arsenite was measured. When the cells were incubated in medium containing $100 \mathrm{mM}$ sodium arsenite for $4 \mathrm{~h}$, the accumulation of arsenite in PC-9/CDDP cells was increased 1.7-fold compared with the accumulation demonstrated by PC-9 cells (Fig. 1). When the cisplatin accumulation in the cells was determined under the same conditions, the cisplatin concentration in the PC-9/CDDP cells was found to be markedly decreased, demonstrating an accumulation of $20 \%$ of the cisplatin found in the parental PC-9 cells (data not shown).

Analysis of the GSH concentration and activity of GSH-associated enzymes. GS-X pump systems are an important mechanism by which metals, including cisplatin and arsenite, are transported $(17,18)$. To investigate the molecular basis for the decreased accumulation of arsenite, the GS-X pump systems were analyzed, including cellular GSH levels, activity of the GSH synthesis rate-limiting enzyme $\gamma$-GCS, GST activity and expression of the ABC transporters. Cellular GSH levels in PC-9/CDDP cells were $65 \%$ of those measured in the parental PC-9 cell line. $\gamma$-GCS activity was also downregulated in PC-9/CDDP cells, where it was $67 \%$ of the activity measured in PC-9 cells. GST activity was also extremely decreased to $\sim 4.0 \%$ of the activity observed in the parental 


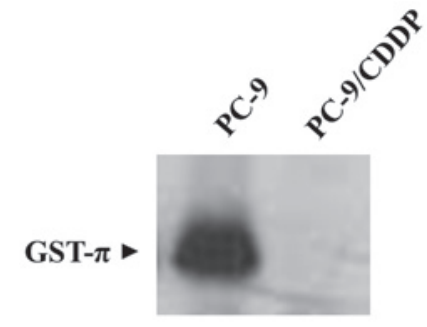

Figure 2. Expression of the GST- $\pi$ protein in the cisplatin-resistant PC-9/CDDP cell line. The GST- $\pi$ protein levels in the parental PC-9 and cisplatin-resistant PC-9/CDDP cells were determined by western blot analysis. GST- $\pi$, glutathione- $S$-transferase- $\pi$.

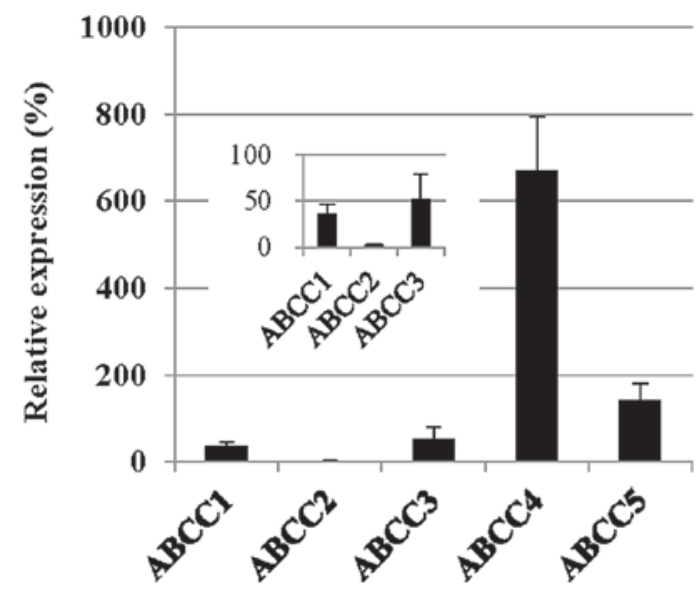

Figure 3. Expression levels of ABCC transporter mRNA in PC-9/CDDP and PC-9 cell lines. The relative expression of ABCC transporter mRNA in PC-9 and PC-9/CDDP cells was determined by quantitative real-time polymerase chain reaction, which was performed using TaqMan probes. GAPDH mRNA was also quantified and used to normalize the mRNA levels of the transporters. Each bar represents the mean \pm standard deviation of triplicate independent experiments. $\mathrm{ABCC}$, adenosine triphosphate-binding cassette subfamily C; ABCC1-5, ABCC, members 1-5.

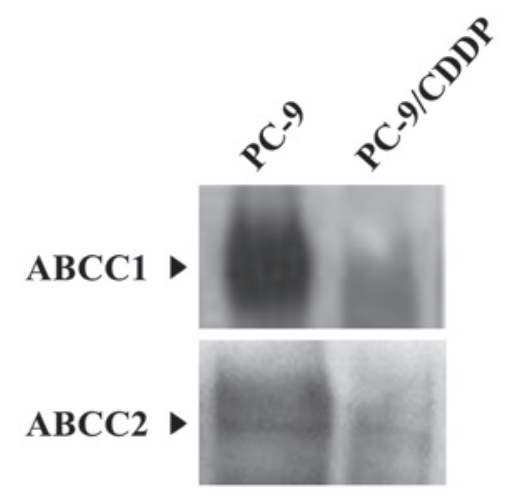

Figure 4. Protein expression levels of the GS-X pump (ABCC1 and 2) in the plasma membrane were downregulated in the cisplatin-resistant PC-9/CDDP cell line. The ABCC1 and $\mathrm{ABCC} 2$ protein levels in the PC-9 and PC-9/CDDP cells were determined by western blot analysis. Plasma membrane fractions were probed with anti-ABCC1 or anti-ABCC2 monoclonal antibodies. $\mathrm{ABCC} 1$, adenosine triphosphate-binding cassette subfamily $\mathrm{C}$, member 1; $\mathrm{ABCC} 2, \mathrm{ABCC}$, member 2.

cell line (Table II). The protein expression of GST- $\pi$ was also downregulated in PC-9/CDDP cells compared with the parental cell line (Fig. 2).
Analysis of the expression of ABCC transporters. It has previously been indicated that arsenite is a substrate for the GS-X pump, as a GS-As conjugate (11). As shown in Fig. 3, the expression of $\mathrm{ABCC} 1,2$ and 3 was downregulated in the PC-9/CDDP cells, with mRNA expression levels of 36.0, 2.7 and $51.9 \%$ of the level measured in the parental PC- 9 cell line, respectively. The protein levels of $\mathrm{ABCC} 1$ and 2 in the plasma membrane were also decreased in the PC-9/CDDP cells compared with the parental cells. In western blot analysis, only faint bands corresponding to $\mathrm{ABCC} 1$ and 2 were obtained from the fractions derived from the PC-9/CDDP plasma membrane (Fig. 4).

Sensitivity of the cisplatin-resistant lung cancer PC-9/CDDP and PC-14/CDDP cell lines to ATO. As aforementioned, the cisplatin-resistant PC-9/CDDP cell line was revealed to demonstrate hypersensitivity to arsenite. Additionally, the cytotoxic effect of the arsenite-containing chemotherapeutic agent ATO was examined in the cisplatin-resistant NSCLC PC-9/CDDP and PC-14/CDDP cell lines.

The findings revealed that the PC-9/CDDP and PC-14/CDDP cell lines each demonstrated a 3 -fold increase in sensitivity to ATO compared with the PC-9 and PC-14 parental cell lines (Table III).

\section{Discussion}

Cisplatin is a coordination compound of platinum. Certain cisplatin-resistant cell lines demonstrate cross-resistance to metals that include cadmium, antimony and arsenite (19). The identification of cross-resistant metals aids the evaluation of the mechanism underlying cisplatin resistance and metal transport systems. Previously, cisplatin-resistant cell lines were developed from metallothionine knockout (MT-KO) cells, and these cell lines demonstrated cross-resistance to arsenite and decreased arsenite accumulation (20). These results indicate the presence of a mechanism by which arsenite and cisplatin resistance in MT-KO cell lines depends on the same transport systems.

By contrast, an in vitro study has revealed that the combined administration of cisplatin and ATO demonstrates a synergistic inhibitory effect on the growth of various cancer cell lines, including squamous carcinoma, ovarian cancer and small cell lung cancer cells (7-9,21).

In the present study, the cisplatin-resistant PC-9/CDDP cell line was revealed to be hypersensitive to arsenite. A marked decrease in the accumulation of arsenite was also observed in the PC-9/CDDP cells. These results indicate that the transport systems for cisplatin and arsenite are independent in PC-9 cells. Consequently, hypersensitivity to arsenite in PC-9/CDDP cells appears to be dependent on the cellular accumulation of arsenite.

It has previously been reported that the mechanisms of arsenite accumulation are mediated by transporter systems, influx transporters, including the aquaglyceroporins aquaporin (AQP)7 and AQP9 (22), and efflux transporters, such as $\mathrm{ABCC} 1$ and $\mathrm{ABCC} 2$ (11). Although the cisplatin uptake transporter was not identified in the present cell lines, the transporter of cisplatin in PC-9/CDDP cells may not contribute to the uptake of arsenite. 
The present results clearly reveal that GSH and GS-X pump-associated factors, including GST activity and ABC transporter expression, were downregulated in PC-9/CDDP cells. GSH and GST are important for the formation of the arsenic-glutathione conjugate, which is a good substrate for $\mathrm{ABCC} 1$ and $\mathrm{ABCC} 2$.

In conclusion, ATO, an approved drug, potentially possesses sensitivity for cisplatin-resistant NSCLC cell lines. The mechanism of ATO hypersensitivity is dependent on the downregulation of GS-X pump systems, particularly GSH, GST, ABCC1 and ABCC2.

The aforementioned findings indicate that ATO has the potential to treat cisplatin resistance in NSCLC and that $\mathrm{ABCC} 1$ and $\mathrm{ABCCC} 2$ are important biomarkers when considering a chemotherapeutic regimen containing ATO for the treatment of cisplatin-resistant NSCLC.

\section{Acknowledgements}

This study was supported by the Japan Society for the Promotion of Science (JSPS) Grants-in-Aid for Scientific Research KAKENHI (grant nos., 20790147, 22790173 and 24590218).

\section{References}

1. Dasari S and Tchounwou PB: Cisplatin in cancer therapy: Molecular mechanisms of action. Eur J Pharmacol 740: 364-378, 2014.

2. Nishio K, Nakamura T, Koh Y, Suzuki T, Fukumoto H and Saijo N: Drug resistance in lung cancer. Curr Opin Oncol 11: 109-115, 1999.

3. Suzuki T, Nishio K and Tanabe S: The MRP family and anticancer drug metabolism. Curr Drug Metab 2: 367-377, 2001.

4. Nordstrom DK: Public health. Worldwide occurrences of arsenic in ground water. Science 296: 2143-2145, 2002.

5. Rao Y, Li R and Zhang D: A drug from poison: How the therapeutic effect of arsenic trioxide on acute promyelocytic leukemia was discovered. Sci China Life Sci 56: 495-502, 2013.

6. Lengfelder E, Hofmann WK and Nowak D: Impact of arsenic trioxide in the treatment of acute promyelocytic leukemia. Leukemia 26: 433-442, 2012

7. Zhang N, Wu ZM, McGowan E, Shi J, Hong ZB, Ding CW, Xia P and Di W: Arsenic trioxide and cisplatin synergism increase cytotoxicity in human ovarian cancer cells: Therapeutic potential for ovarian cancer. Cancer Sci 100: 2459-2464, 2009.

8. Helm CW and States JC: Enhancing the efficacy of cisplatin in ovarian cancer treatment-could arsenic have a role. J Ovarian Res 2: 2, 2009 .
9. Zheng CY, Lam SK, Li YY, Fong BM, Mak JC and Ho JC: Combination of arsenic trioxide and chemotherapy in small cell lung cancer. Lung Cancer 82: 222-230, 2013.

10. Szakács G, Paterson JK, Ludwig JA, Booth-Genthe C and Gottesman MM: Targeting multidrug resistance in cancer. Nat Rev Drug Discov 5: 219-234, 2006.

11. Leslie EM: Arsenic-glutathione conjugate transport by the human multidrug resistance proteins (MRPs/ABCCs). J Inorg Biochem 108: 141-149, 2012.

12. Hong WS, Saijo N, Sasaki Y, Minato K, Nakano H, Nakagawa K, Fujiwara Y, Nomura K and Twentyman PR: Establishment and characterization of cisplatin-resistant sublines of human lung cancer cell lines. Int J Cancer 41: 462-467, 1988.

13. Uemura M, Suzuki T, Nishio K, Chikuma M and Komeda S: An in vivo highly antitumor-active tetrazolato-bridged dinuclear platinum (II) complex largely circumvents in vitro cisplatin resistance: Two linkage isomers yield the same product upon reaction with 9-ethylguanine but exhibit different cytotoxic profiles. Metallomics 4: 686-692, 2012.

14. Mukai Y, Togawa T, Suzuki T, Ohata K and Tanabe S: Determination of homocysteine thiolactone and homocysteine in cell cultures using high-performance liquid chromatography with fluorescence detection. J Chromatogr B Analyt Technol Biomed Life Sci 767: 263-268, 2002.

15. Habig WH, Pabst MJ, Fleischner G, Gatmaitan Z, Arias IM and Jakoby WB: The identity of glutathione S-transferase B with ligandin, a major binding protein of liver. Proc Natl Acad Sci USA 71: 3879-3882, 1974.

16. Yoshikawa M, Ikegami Y, Hayasaka S, Ishii K, Ito A, Sano K, Suzuki T, Togawa T, Yoshida H and Soda H: Novel camptothecin analogues that circumvent ABCG2-associated drug resistance in human tumor cells. Int J Cancer 110: 921-927, 2004.

17. Kurokawa H, Ishida T, Nishio K, Arioka H, Sata M, Fukumoto H, Miura M and Saijo N: Gamma-glutamylcysteine synthetase gene overexpression results in increased activity of the ATP-dependent glutathione S-conjugate export pump and cisplatin resistance. Biochem Biophys Res Comm 216: 258-264, 1995.

18. Ishikawa T, Bao JJ, Yamane Y, Akimaru K, Frindrich K, Wright CD and Kuo MT: Coordinated induction of MRP/GS-X pump and gamma-glutamylcysteine synthetase by heavy metals in human leukemia cells. The Journal of biological chemistry 271: 14981-14988, 1996.

19. Naredi P, Heath DD, Enns RE and Howell SB: Cross-resistance between cisplatin, antimony potassium tartrate and arsenite in human tumor cells. J Clin Invest 95: 1193-1198, 1995.

20. Suzuki T, Ohata S, Togawa T, Himeno S and Tanabe S: Arsenic accumulation decreased in metallothionein null Cisplatin-resistant cell lines. J Toxicol Sci 32: 321-328, 2007.

21. Nakaoka T, Ota A, Ono T, Karnan S, Konishi H, Furuhashi A Ohmura Y, Yamada Y, Hosokawa Y, Kazaoka Y, et al: Combined arsenic trioxide-cisplatin treatment enhances apoptosis in oral squamous cell carcinoma cells. Cell Oncol (Dordr) 37: 119-129, 2014.

22. Liu Z, Shen J, Carbrey JM, Mukhopadhyay R, Agre P and Rosen BP: Arsenite transport by mammalian aquaglyceroporins AQP7 and AQP9. Proc Natl Acad Sci USA 99: 6053-6058, 2002. 